\title{
Clinical characteristics of the autumn-winter type scrub typhus cases in south of Shandong province, northern China
} Yun-Xi Liu*1, Dan Feng2, Ji-Jiang Suo1, Yu-Bin Xing1, Gang Liu ${ }^{3}$, Li-Hua Liu², Hong-Ju Xiao ${ }^{3}$, Ning Jia1, Yan Gao${ }^{1}$, Hong Yang ${ }^{4}$, Shu-Qing Zuo ${ }^{4}$, PanHe Zhang ${ }^{4}$, Zhong-Tang Zhao ${ }^{5}$, Jing-Si Min ${ }^{6}$, Pei-Tian Feng ${ }^{7}$, Shu-Bin Ma ${ }^{8}$, Song Liang*9 and Wu-Chun Cao*4

\author{
Address: ${ }^{1}$ Department of Nosocomial Infection Management and Disease Control, Institute of Hospital Management, Chinese People's Liberation \\ Army General Hospital, Chinese People's Liberation Army Postgraduate Medical School, Beijing 100853, PR China, ${ }^{2}$ Department of Health \\ Statistics, Institute of Hospital Management, Chinese People's Liberation Army General Hospital, Chinese People's Liberation Army Postgraduate \\ Medical School, Beijing 100853, PR China, ${ }^{3}$ Department of Febrile Disease, Chinese People's Liberation Army General Hospital, Chinese People's \\ Liberation Army Postgraduate Medical School, Beijing 100853, PR China, ${ }^{4}$ State Key Laboratory of Pathogen and Biosecurity, Beijing Institute of \\ Microbiology and Epidemiology, Beijing 100071, PR China, ${ }^{5}$ School of Public Health, Shandong University, Jinan 250012, PR China, \\ ${ }^{6}$ Department of Internal Medicine, Wanggou Township Hospital, Feixian County, Shandong Province 273408, PR China, ${ }^{7}$ Department of Internal \\ Medicine, Shangye Township Hospital, Feixian County, Shandong Province 273401, PR China, ${ }^{8}$ Department of Internal Medicine, Fangcheng \\ Township Hospital, Feixian County, Shandong Province 273409, PR China and ${ }^{9}$ College of Public Health, The Ohio State University, Columbus, \\ Ohio 43210, USA \\ Email: Yun-Xi Liu* - Yunxiliu6511@yahoo.com.cn; Dan Feng - fddd@263.net; Ji-Jiang Suo - si191919@yahoo.com.cn; Yu- \\ Bin Xing - quanjun2000@yahoo.com.cn; Gang Liu - liugangfever@sohu.com; Li-Hua Liu - llh150@vip.sina.com; Hong- \\ Ju Xiao - xiaohongjufever@sohu.com; Ning Jia - Jianing@263.net; Yan Gao - Gaoy301@sina.com; Hong Yang - anni_yang@yahoo.com; Shu- \\ Qing Zuo - Zuoshq@yahoo.com.cn; Pan-He Zhang - juntianz@yahoo.com.cn; Zhong-Tang Zhao - ztzhao@sdu.edu.cn; Jing- \\ Si Min - jingsiminfx@sohu.com; Pei-Tian Feng - peitianfengfx@sohu.com; Shu-Bin Ma - shubinmafx@sohu.com; \\ Song Liang* - sliang@cph.osu.edu; Wu-Chun Cao* - caowc@nic.bmi.ac.cn \\ * Corresponding authors
}

Published: 4 June 2009

BMC Infectious Diseases 2009, 9:82 doi:10.1 186/1471-2334-9-82
Received: 18 October 2008

Accepted: 4 June 2009

This article is available from: http://www.biomedcentral.com/I47/-2334/9/82

(c) 2009 Liu et al; licensee BioMed Central Ltd.

This is an Open Access article distributed under the terms of the Creative Commons Attribution License (http://creativecommons.org/licenses/by/2.0), which permits unrestricted use, distribution, and reproduction in any medium, provided the original work is properly cited.

\begin{abstract}
Background: Before 1986, scrub typhus was only found endemic in southern China. Because human infections typically occur in the summer, it is called "summer type". During the autumnwinter period of 1986, a new type of scrub typhus was identified in Shandong and northern Jiangsu province of northern China. This newly recognized scrub typhus was subsequently reported in many areas of northern China and was then called "autumn-winter type". However, clinical characteristics of associated cases have not been reported.
\end{abstract}

Methods: From 1995 to 2006, all suspected scrub typhus cases in five township hospitals of Feixian county, Shandong province were enrolled. Indirect immunofluorescent assay (IFA) was used as confirmatory serodiagnosis test. Polymerase chain reaction (PCR) connected with restriction fragment length polymorphism (RFLP) and sequence analyses were used for genotyping of $O$. tsutsugamushi DNAs. Clinical symptoms and demography of confirmed cases were analyzed.

Results: A total of 480 scrub typhus cases were confirmed. The cases occurred every year exclusively between September and December with a peak occurrence in October. The case numbers were relatively higher in 1995, 1996, 1997, and 2000 than in other years. $57.9 \%$ of cases were in the group aged $2 \mathrm{I}-50$. More cases occurred in male (56\%) than in female (44\%). The 
predominant occupational group of the cases was farmers (85.0\%). Farm work was reported the primary exposure to infection in $67.7 \%$ of cases. Fever, rash, and eschar were observed in $100.0 \%$, $90.4 \%$, and $88.5 \%$ of cases, respectively. Eschars formed frequently on or around umbilicus, abdomen areas, and front and back of waist (34.1\%) in both genders. Normal results were observed in $88.7 \%$ (WBC counts), 84.5\% (PLT counts), and $89.7 \%$ (RBC counts) of cases, respectively. Observations from the five hospitals were compared and no significant differences were found.

Conclusion: The autumn-winter type scrub typhus in northern China occurred exclusively from September to December with a peak occurrence in October, which was different from the summer type in southern China. In comparison with the summer type, complications associated with autumn-winter type scrub typhus were less severe, and abnormalities of routine hematological parameters were less obvious.

\section{Background}

Scrub typhus is a rickettsial disease caused by Orientia tsutsugamushi [1], which is transmitted to humans through infected chigger mites. Scrub typhus is widely distributed in Southeast Asia and the Pacific Rim including China $[1,2]$. When the rickettsia is transmitted through the bite of an infected mite to human, it begins to proliferate at the bite site and a characteristic skin lesion, known as an eschar, is formed. The pathogen then spreads systemically via the hematogenous and lymphogenous routes. Infected people develop various systemic symptoms and reactions including fever, cutaneous rash, lympadenopathy, elevations of C-reacting protein (CRP) and liver enzymes [2-4].

Prior to 1986, scrub typhus was only found endemic in southern China (south of the Yangtse River, or to the south of $31^{\circ}$ north latitude), including 11 provinces (e.g., Guangdong, Hainan, Guangxi, Fujian, Zhejiang, Yunnan, Hunan province). Because human infections typically occur between March and November with a peak occurrence between June and August in the summer [5-7], so the scrub typhus is also called "summer type" scrub typhus, which is transmitted by the Leptotrombidium deliense mite [5-8]. The reservoir hosts are rodents mainly including Rattus losea, $R$. flavipectus, and Apodemus agrarius [5-7]. Major serotypes of summer type scrub typhus in many areas of southern China were Karp, Gilliam, and Kato types [5-7,9-11]. In China, genotypes of scrub typhus have not been systematically studied until recently $[6,7,9]$. However, the genotyping results obtained in Guangdong, Fujian, Hainan province of southern China revealed that Karp types were the key genotypes of summer type scrub typhus in these areas [12-14]. The summer type scrub typhus is caused by a relatively more virulent strain of $O$. tsutsugamushi $[5,7]$. Human cases caused by the summer type scrub typhus have common clinical features including fever, cutaneous rash, eschar and local lympadenopathy, and the associated complications were typically severe [5-7].
During the autumn-winter period of 1986, some residents in Mengyin county, south of Shandong province [15], and Dongtai, northern Jiangsu province [16] (both located in north of the Yangtse River, or to the north of $31^{\circ}$ north latitude) developed an unknown fever, which was later identified to be caused by scrub typhus. As cases associated with this type of scrub typhus occurred from September to December with an occurrence peak in October, it was called "autumn-winter type". This type of scrub typhus was subsequently reported in many regions of northern China including Tianjing, Shanxi province, Hebei province, and Henan province $[5-7,17,18]$. The autumn-winter type scrub typhus is caused by a less virulent strain of O. tsutsugamushi and transmitted by L. scutellare mite $[5-7,19]$. The reservoir hosts are A. agrarius, Cricetulus triton, and R. norvegicus [5-7,19]. Although Gilliam types were identified by IFA as the key serotypes of autumn-winter type scrub typhus in many areas of northern China [5-7,14,19], the genotyping results acquired in Shandong, northern Jiangsu province in northern China showed that Kawasaki types were the key genotypes of this type of scrub typhus in the study areas (the genotypes in other areas were not studied or undetermined) [14,20$22]$. Since 1986 , people infected with autumn-winter type scrub typhus have been increasing in many areas of northern China $[5-7,17,18]$. However, clinical characteristics of the newly recognized scrub typhus have not been studied. In this study, we summarize the clinical characteristics of human infections by autumn-winter type scrub typhus in Feixian county, south of Shandong province, northern China.

\section{Methods \\ The study site}

The study was conducted from 1995 to 2006 in Feixian county $\left(117^{\circ} 11^{\prime}-118^{\circ} 18^{\prime} \mathrm{E}, 35^{\circ} 01^{\prime}-35^{\circ} 33^{\prime} \mathrm{N}\right)$, which is located in a mountainous area in the south of Shandong province (Figure 1). The northwestern parts of Feixian county belong to the range of Meng Mountains, while the south parts belong to the range of Ni Mountains respectively. The northeastern and central parts of Feixian 


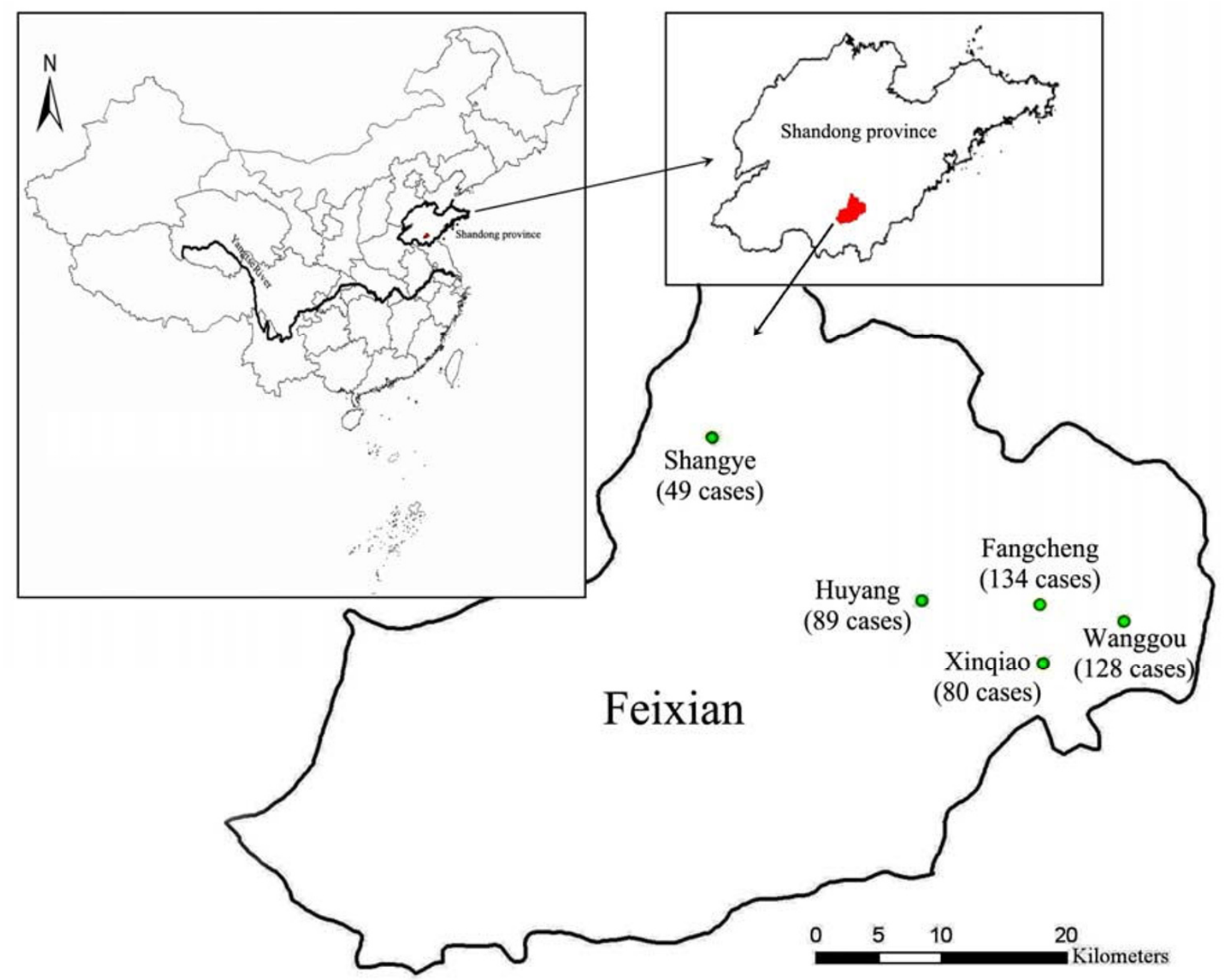

Figure I

Distribution of the study sites. The map shows location of the study sites and distribution of confirmed cases in the five towns of Feixian county in southern Shandong province, China. The numbers in parentheses denote the total number of cases in each town during the 12-year study period.

county are flatlands in front of mountain within its boundaries, and located in temperate and sub-humid areas with distinct four seasons, the county has an annual average temperature $13.4^{\circ} \mathrm{C}$ with the highest average $26.3^{\circ} \mathrm{C}$ in July and lowest $-1.8^{\circ} \mathrm{C}$ in January [23]. We selected five coadjacent township hospitals (Fangcheng, Wanggou, Huyang, Xinqiao, and Shangye), which serve for about 260,000 local residents in the surrounding 22 $\mathrm{km}^{2}$ area in the northeast of Feixian county, where scrub typhus was first reported in 1988 and became endemic since then during the autumn-winter seasons [5-7].

\section{Criteria for case selections}

Between 1995 and 2006, patients who were admitted to the five township hospitals, had acute undifferentiated fever and clinical manifestations indicative of scrub typhus were enrolled in the study as suspected cases. The clinical manifestations included acute fever, eschars, local lympadenopathy, and maculopapular rashes on the trunk and proximal limbs. For each enrolled case, information on past activities (e.g., farming, playing, and recreation in the farm field where rodents were present) within 1-3 weeks (this time window is the incubation period of the autumn-winter type scrub typhus) prior to his/her illness were recorded. After informed consent was obtained, acute and convalescent blood samples and eschars from 
suspected cases were collected for subsequent IFA and PCR analyses. People with a history of known chronic medical problems or illness were excluded in the study. The study was approved by the Ethical and Scientific Review Subcommittee of the Ministry of Public Health of China.

\section{Confirmatory diagnosis}

For each suspected case, the serum was assayed by IFA for detecting the IgM and IgG antibodies against pooled Karp, Kato, and Gilliam strains of $O$. tsutsugamushi antigens $[5,7,14,19]$. Serotyping of each positive serum was carried out as previously described $[5,7,14,22]$. A confirmed scrub typhus case was defined as (1) IgG titer $\geq 4$-fold increase in paired serum specimens; and (2) IgM titer $\geq$ $1: 80$ or IgG titer $\geq 1: 400$ in a single serum sample, $[5,24$ 26]. PCR method became available in 2001 and was used for detection and genotyping of O. tsutsugamushi connected with RFLP and nucleotide sequences analyses since then $[21,22,27]$.

\section{Data collection}

Questionnaire we developed were administered by physicians working in the five township hospitals. The questionnaire were designed to include (1) demography: age, sex, education level, and occupation; (2) epidemiologic data: symptom onset date, residence place, and activities possibly associated with scrub typhus infection within 13 weeks prior to onset of symptoms; (3) clinical signs and symptoms: fever, eschar, rash, lymphadenopathy, and others; (4) routine hematological examination: white blood cell (WBC) counts, platelet (PLT) counts, red blood cell (RBC), hemoglobin (HGB) and hematocrit (HCT); and (5) diagnosis: results of IFA and PCR analyses. All collected data were double-entered in spreadsheet for subsequent statistical analyses.

\section{Statistical analyses}

Statistical analyses were performed using the Chi-square test (or Fisher's exact test) for categorical data. A difference was considered statistically significant if the $P$ value was less than 0.05 . Ranges were indicated by IQR (interquartile range).

\section{Results \\ Confirmed cases of scrub typhus}

From 1995 to 2006, a total of 678 suspected cases were enrolled in the study. Based on results from IFA examination, 280 scrub typhus cases were confirmed based on a fourfold or greater increase between paired serum specimens; 86 cases were confirmed by having an IgM titer $\geq$ $1: 80$ in a single serum sample; and 114 cases were confirmed by having an IgG titer $\geq 1: 400$ in a single serum sample. The serotypes of the 480 IFA-positive samples were all Gilliam types. The rest 198 cases were non-scrub typhus cases as identified by IFA. 15 isolates were obtained from 40 IFA-positive cases during the study period $[19,21,22]$. Figure 1 showed location of the study area and distribution of the 480 cases in the five towns: 134 (27.9\%) cases in Fangcheng, 128 (26.7\%) cases in Wanggou, 89 (18.5\%) cases in Huyang, 80 (16.7\%) cases in Xinqiao, and 49 (10.2\%) cases in Shangye.

Among the IFA-positive samples collected from 2001 to 2006, 34 out of the 45 acute stage blood samples of cases were PCR-positive. Ten eschars from 10 cases whose serum samples were IFA-positive and blood samples were PCR-positive were also tested positive by PCR. In addition, 15 isolates were tested positive by PCR [21,22]. The genotypes of the above 59 PCR-positive samples were similar to Kawasaki strain with one mutation of restriction endonuclease Hha-site (details were discussed elsewhere, see references $21,22,27)$.

Chloramphenicol was administered orally at dosages of 1.5-2.5 g 4×/day for 4-5 days for adult cases and 25-50 $\mathrm{mg} / \mathrm{kg} 4 \times /$ day for 4 to 5 days for pediatric cases, respectively. The median interval to defervescenct-period after chloramphenicol therapy was 2 days (range 1-5 d). All of the cases defervesced after treatment, except one case (male, 70-year-old) who was initially misdiagnosed by the country infirmary. The case was sent to Shangye township hospital with serious multiple system/organ failures, and eventually died after all efforts for rescuing him failed.

\section{Seasonal distribution of confirmed cases}

In the study areas over the past 12 years, scrub typhus cases occurred exclusively every year from the period of day 11-20, September to the period of day 1-10, December, and seasonal distributions of the cases were similar from 1995 to 2006 (Table 1). During the study period, no case was reported before September and after December and peaks of cases occurrences were all in October, followed by a sharp drop in case numbers. The case numbers were relatively higher in 1995, 1996, 1997, and 2000 than in other years.

\section{Sex and age distributions of confirmed cases}

Of 480 confirmed cases, 56\% (269/480) were male patients and 44\% (211/480) were female, respectively. The percentage of male cases was higher than that of females in all groups except for the group aged 31-40 $\left(\chi^{2}\right.$ $=42.4, \mathrm{P}<0.001) .278(57.9 \%)$ cases were in the group aged $21-50$, followed by $102(21.3 \%), 79(16.5 \%)$ and 21 $(4.4 \%)$ cases in the group aged $51-70,0-20$, and $\geq 71$, respectively. The youngest and oldest cases were 1.5 and 81 years old, respectively (Table 2 ). 
Table I: Yearly and seasonal distribution of confirmed cases

\begin{tabular}{|c|c|c|c|c|c|c|c|c|c|c|c|c|c|}
\hline \multirow[t]{2}{*}{ Year } & \multicolumn{3}{|c|}{ September } & \multicolumn{3}{|c|}{ October } & \multicolumn{3}{|c|}{ November } & \multicolumn{3}{|c|}{ December } & \multirow[t]{2}{*}{ Total (\%) } \\
\hline & $1-10$ & $11-20$ & $21-30$ & $1-10$ & $11-20$ & $2|-3|$ & $1-10$ & $11-20$ & $21-30$ & $1-10$ & $11-20$ & $2|-3|$ & \\
\hline 1995 & 0 & 0 & 1 & 10 & 44 & 41 & 19 & 4 & 0 & 0 & 0 & 0 & $119(24.8)$ \\
\hline 1996 & 0 & 0 & 3 & 4 & 8 & 20 & 12 & 10 & I & 0 & 0 & 0 & $58(12.1)$ \\
\hline 1997 & 0 & 0 & 3 & 8 & 20 & 10 & 7 & 2 & 0 & 0 & 0 & 0 & $50(10.4)$ \\
\hline 1998 & 0 & 0 & 0 & 6 & 2 & 19 & 5 & 0 & 0 & 0 & 0 & 0 & $32(6.7)$ \\
\hline 1999 & 0 & 0 & 0 & 7 & 11 & 6 & 5 & 0 & 0 & 0 & 0 & 0 & $29(6.0)$ \\
\hline 2000 & 0 & 0 & 0 & 8 & 15 & 5 & 7 & 5 & 0 & 0 & 0 & 0 & $40(8.3)$ \\
\hline 2001 & 0 & 0 & 0 & 8 & 10 & 2 & 1 & 0 & 0 & 0 & 0 & 0 & $21(4.4)$ \\
\hline 2002 & 0 & I & 2 & 9 & 13 & 3 & 3 & 4 & 0 & 0 & 0 & 0 & $35(7.3)$ \\
\hline 2003 & 0 & I & 0 & 2 & 3 & 7 & 2 & 0 & 0 & I & 0 & 0 & $16(3.3)$ \\
\hline 2004 & 0 & 0 & 2 & 0 & 11 & 5 & 5 & 1 & 0 & 0 & 0 & 0 & $24(5.0)$ \\
\hline 2005 & 0 & 0 & 1 & 5 & 4 & 16 & 6 & 0 & 1 & 0 & 0 & 0 & $33(6.9)$ \\
\hline 2006 & 0 & 0 & 0 & I & 6 & 11 & 4 & 0 & 1 & 0 & 0 & 0 & $23(4.8)$ \\
\hline Total & 0 & 2 & 12 & 68 & 147 & 145 & 76 & 26 & 3 & 1 & 0 & 0 & 480 \\
\hline$(\%)$ & 0 & $(0.4)$ & $(2.5)$ & $(\mid 4.2)$ & $(30.6)$ & $(30.2)$ & $(15.8)$ & (5.4) & $(0.6)$ & $(0.2)$ & $(0)$ & (0) & $(100)$ \\
\hline
\end{tabular}

\section{Occupation and activities possibly connected with infection}

The predominant occupational group of the cases was farmers $(85.0 \%)$, followed by pupils $(10.4 \%)$, and preschool children (4.2\%). The percentage of male cases was higher than that of females in the above three groups $\left(\chi^{2}\right.$ $=28.4, P<0.001)$. The human activities possibly associated with infection included farm work (325 cases, $67.7 \%$ ), playing on grassland (65 cases, $13.5 \%$ ), recreation (20 cases, 4.2\%), and housework (25 cases, 5.2\%). The percentage of male cases was higher than that of females in all categories except the housework group $\left(\chi^{2}=\right.$ 119.6, $P<0.001$, Table 2).

\section{Clinical symptoms}

Table 3 summarize the symptoms and signs of all 480 confirmed cases at the time of admission to hospitals with additional 274 cases previously collected from 1996 to 2005 in Shaoguan, Guangdong province, southern China [28], where the "summer type" scrub typhus was endemic. In present study, cases manifested with the common clinical symptoms of scrub typhus: fever $(100.0 \%$ of confirmed cases), rash (90.4\%), eschar (88.5\%), and regional

Table 2: Age and occupational distributions and activities ( $1-3$ weeks prior to symptom appearance) of confirmed cases

\begin{tabular}{llll}
\hline & & Cases (\%) & (Male: Female, \%) \\
\hline Age (Years) & & & $(76: 24)$ \\
& $0-10$ & $46(9.6)$ & $(79: 21)$ \\
& $11-20$ & $33(6.9)$ & $(51: 49)$ \\
& $21-30$ & $78(16.3)$ & $(46: 54)$ \\
& $31-40$ & $99(20.6)$ & $(56: 44)$ \\
& $41-50$ & $101(21.0)$ & $(55: 45)$ \\
& $51-60$ & $47(9.8)$ & $(53: 47)$ \\
& $61-70$ & $55(11.5)$ & $(52: 48)$ \\
Occupation & $\geq 71$ & $21(4.4)$ & $(75: 25)$ \\
& Children at preschool age & & $(78: 22)$ \\
& Pupils & $20(4.2)$ & $(52: 48)$ \\
Fctivities & Farmers & $50(10.4)$ & $(50: 50)$ \\
& Others & $408(85.0)$ & $(53: 47)$ \\
& Farm work & $2(0.4)$ & $(75: 25)$ \\
& Play on grassland & & $(65: 35)$ \\
& Recreation & $325(67.7)$ & $(8: 92)$ \\
& Housework & $65(13.5)$ & $(71: 29)$
\end{tabular}


Table 3: Clinical characteristics of confirmed cases at the time of hospital admission

\begin{tabular}{|c|c|c|c|c|}
\hline Clinical symptoms & & $\begin{array}{l}\text { Present study } \\
N=480(\%)\end{array}$ & $\begin{array}{l}\text { Other study }[28] \\
N=274(\%)\end{array}$ & $P$-value \\
\hline \multicolumn{5}{|c|}{ Typical symptoms of scrub typhus } \\
\hline & Fever & $480(100.0)$ & $274(100.0)$ & -- \\
\hline & $\begin{array}{l}\text { Maculopapular rashes appeared on the trunk and } \\
\text { proximal limbs }\end{array}$ & $434(90.4)$ & $102(37.2)$ & $<0.00 I^{a}$ \\
\hline & Skin eschars & $425(88.5)$ & $240(87.6)$ & 0.697 a \\
\hline & Regional lymphadenopathy & $291(60.6)$ & $248(90.5)$ & $<0.00 \mathrm{I}^{\mathrm{a}}$ \\
\hline \multicolumn{5}{|c|}{ General symptoms of febrile disease } \\
\hline & Chills & $323(67.3)$ & $125(45.6)$ & $<0.00 \mathrm{la}^{\mathrm{a}}$ \\
\hline & Loss of appetite & $398(82.9)$ & $243(88.7)$ & $0.033^{\mathrm{a}}$ \\
\hline & Erythematous flushes on the face, neck and upper bosom & $234(48.8)$ & -- & -- \\
\hline & Myalgia and prostration & $480(100.0)$ & $250(91.2)$ & $<0.00 \mathrm{I}^{\mathrm{a}}$ \\
\hline \multicolumn{5}{|l|}{ Ache } \\
\hline & Headache & $480(100.0)$ & $135(49.3)$ & $<0.00 \mathrm{l}^{\mathrm{b}}$ \\
\hline & Retro-orbital pain & $83(17.3)$ & $188(68.6)$ & $<0.00 \mathrm{la}^{\mathrm{a}}$ \\
\hline \multicolumn{5}{|l|}{ Heart damage } \\
\hline & Electrocardiogram abnormity & $38(7.9)$ & $74(27.0)$ & $<0.00 \mathrm{la}^{\mathrm{a}}$ \\
\hline & Toxic myocarditis & 0 & $57(20.8)$ & $<0.00 \mathrm{I}^{\mathrm{b}}$ \\
\hline \multicolumn{5}{|c|}{ Digestive system damage } \\
\hline & Abdominal pain & $234(48.8)$ & $67(24.5)$ & $<0.00 \mathrm{lb}^{\mathrm{b}}$ \\
\hline & Nausea/vomiting & $210(43.8)$ & $62(22.6)$ & $<0.00 \mathrm{I}^{\mathrm{a}}$ \\
\hline & Hepatosplenomegaly & $84(17.5)$ & $166(60.6)$ & $<0.00 \mathrm{Ib}^{\mathrm{b}}$ \\
\hline & Alimentary tract hemorrhage & 0 & $34(12.4)$ & $<0.001^{b}$ \\
\hline & Pleural fluid, or abdominal dropsy & 0 & $27(9.9)$ & $<0.00 I^{b}$ \\
\hline \multicolumn{5}{|l|}{ Kidney damage } \\
\hline & Flank tenderness & $48(10.0)$ & $86(31.39)$ & $<0.001$ a \\
\hline
\end{tabular}

$\mathbf{a}$ - Chi-square test; $\mathbf{b}$ - Fisher's exact test.

lymphadenopathy (60.6\%). Headache (100.0\%), myalgia and prostration $(100.0 \%)$, loss of appetite $(82.9 \%)$, chills $(67.3 \%)$, abdominal pain $(48.8 \%)$, erythematous flushes $(48.8 \%)$, nausea/vomiting $(43.8 \%)$, retro-orbital pain $(17.3 \%)$, and flank tenderness $(10.0 \%)$ were observed respectively. Hepatosplenomegaly and electrocardiogram abnormity were reported in $84(17.5 \%)$ and $38(7.9 \%)$ cases, respectively. No cases had the severe complications such as toxic myocarditis, alimentary tract hemorrhage, pleural fluid, or abdominal dropsy which were reported in southern China [28].

\section{Location of eschars}

Of 480 confirmed scrub typhus cases, $88.5 \%$ (425/480) exhibited one eschar, of which $89.2 \%(240 / 269)$ was found in male while $87.7 \%$ (185/211) was observed in female patients. None of confirmed cases showed two or more eschars (Table 4).

Eschars formed frequently on or around umbilicus, abdomen areas, and front and back of waist (34.1\%), followed by axilla $(20.9 \%)$, and perineal, inguinal areas, anus, buttock, penis or scrotum/labia $(20.7 \%)$. There were no dif-

Table 4: Eschar distributions on bodies of confirmed scrub typhus cases

\begin{tabular}{|c|c|c|c|c|}
\hline Eschar location & $\begin{array}{l}\text { Total cases } \\
\text { (\%) }\end{array}$ & $\begin{array}{l}\text { Male cases } \\
\text { (\%) }\end{array}$ & $\begin{array}{l}\text { Female cases } \\
\text { (\%) }\end{array}$ & $P$-value \\
\hline Head, face, eye, and neck & $14(3.3)$ & $9(3.8)$ & $5(2.7)$ & 0.549 a \\
\hline Front chest above umbilicus (including breast) & $40(9.4)$ & $10(4.2)$ & $30(16.2)$ & $<\left.0.00\right|^{a}$ \\
\hline Back & II (2.6) & $8(3.3)$ & $3(1.6)$ & $0.362 \mathrm{~b}$ \\
\hline Axilla & $89(20.9)$ & $49(20.4)$ & $40(21.6)$ & $0.762^{\mathrm{a}}$ \\
\hline On or around umbilicus, abdomen areas, and front and back of waist & $145(34.1)$ & $86(35.8)$ & $59(31.9)$ & $0.395^{\mathrm{a}}$ \\
\hline Perineal, inguinal areas, anus, buttock, penis or scrotum/labia & $88(20.7)$ & $54(22.5)$ & $34(18.4)$ & $0.298^{\mathrm{a}}$ \\
\hline Upper extremities & $18(4.2)$ & II (4.6) & $7(3.8)$ & $0.685^{\mathrm{a}}$ \\
\hline Lower extremities & $20(4.7)$ & $13(5.4)$ & $7(3.8)$ & $\left.0.43\right|^{a}$ \\
\hline Total & $425(100.0)$ & $240(100.0)$ & $185(100.0)$ & \\
\hline
\end{tabular}

$\mathbf{a}$ - Chi-square test; $\mathbf{b}$ - Fisher's exact test. 
ferences in the locations of the eschar sites between the sexes, except that the eschar appeared more frequently on the front chest above the umbilicus of the females $(16.2 \%)$ than males $(4.2 \%)\left(\chi^{2}=17.8, P<0.001\right)$.

\section{Routine hematological examinations}

For routine hematological examinations of the confirmed cases at the time of admission, both normal and abnormal (e.g. increased or decreased counts) hematological parameters were recorded. Normal results were observed in $88.7 \%$ (WBC counts), $84.5 \%$ (PLT counts), $89.7 \%$ (RBC counts), 68.8\% (HGB), and $43.9 \%$ (HCT) of cases, respectively, while $10.5 \%, 10.9 \%, 8.4 \%, 12.2 \%$, and $15.1 \%$ of cases showed increased counts for each of these categories, respectively (Table 5).

\section{Discussion}

In this study, we documented occurrence periods for the autumn-winter type scrub typhus cases. The seasonal variation of scrub typhus cases in northern China was similar to that reported in Korea, where human cases of scrub typhus were reported to increase in October and peak in November [29]. This pattern was, however, different from those reported in Japan [30,31] and in southern China [57]. In Japan, a bimodal pattern of occurrence of cases one in spring and another in autumn-winter (the latter being similar to the autumn-winter type reported in current study) $[30,31]$ - was reported. Yet in southern China, scrub typhus, caused by the summer type, was endemic with a single peak of infections occurring in summer [5-
$7,28]$. Because humans are infected through bites of the larva of the chigger mites, seasonal variations in scrub typhus infections may be in part due to seasonal fluctuations of the larval chigger mites as well as their rodent hosts. In Japan, the bimodal pattern of occurrence of cases was reported to relate to population dynamics of the two different species of chigger mites. Cases occurred in the autumn-winter period in many areas of Japan were mainly due to L. scutellare, while cases in the spring period in western Japan were caused primarily through $L$. pallidum $[30,31]$. In southern China, seasonal variations in scrub typhus infections were commonly consistent with seasonal variations in population of $L$. deliense [5-8]. The authors conducted a survey on rodent dynamics between May 1995 and April 1996 in the same study area and found that the population abundance of A. agrarius, the most abundant rodent species in the enzootic area, showed a bimodal pattern in a year - the first in July and the second in October. O. tsutsugamushi was isolated from A. agrarius, which was confirmed as the host for scrub typhus in the areas $[19,32]$. Seasonal variations in chigger mites on the field rodents were also observed in this oneyear study, showing that $L$. scutellare first appeared during the period of day 11-20 of September (the chigger index was 13.51) and its abundance then increased sharply in October (the chigger index increased to 22.68). The chigger population peaked during the period of day 11-20 of November (the chigger index peaked to 36.56) and dropped substantially in December (the chigger index in December was 0.47). In addition, O. tsutsugamushi was

Table 5: Routine laboratory findings of confirmed cases at the time of hospital admission to hospitals

\begin{tabular}{|c|c|c|c|c|}
\hline Findings & $\begin{array}{l}\text { Median } \\
\text { (IQR) }\end{array}$ & $\begin{array}{l}\text { Present study } \\
N=478(\%)\end{array}$ & $\begin{array}{l}\text { Other study [28] } \\
N=274(\%)\end{array}$ & $P$-value \\
\hline WBC counts $\left(10^{9} / \mathrm{L}\right)$ & $6.7(5.4-9.3)$ & & & \\
\hline Increase $(>10.0)$ & & $50(10.5 \%)$ & $90(32.9)$ & $<0.00 I^{a}$ \\
\hline Normal $(4.0-10.0)$ & & $424(88.7 \%)$ & $110(40.1)$ & \\
\hline Decrease $(<4.0)$ & & $4(0.8 \%)$ & $74(27.0)$ & \\
\hline PLT counts(109/L) & 143 (120-206) & & & \\
\hline Increase $(>300)$ & & $52(10.9 \%)$ & --- & $<\left.0.00\right|^{a}$ \\
\hline Normal (100-300) & & $404(84.5 \%)$ & 134 (48.9)* & \\
\hline Decrease $(<100)$ & & $22(4.6 \%)$ & $140(5 \mid .1)$ & \\
\hline RBC counts $\left(10^{12} / \mathrm{L}\right)$ & $5.1(4.4-5.3)$ & & & \\
\hline Increase $(>5.5)$ & & $40(8.4 \%)$ & --- & \\
\hline Normal (3.5-5.5) & & $429(89.7 \%)$ & & \\
\hline Decrease $(<3.5)$ & & $9(1.9 \%)$ & & \\
\hline $\operatorname{HGB}(g / L)$ & $149(119-151)$ & & & \\
\hline Increase $(>160)$ & & $58(12.2 \%)$ & --- & \\
\hline Normal (II0-160) & & $329(68.8 \%)$ & --- & \\
\hline Decrease $(<110)$ & & 91 (19.0\%) & & \\
\hline HCT (L/L) & $0.4(0.3-0.5)$ & & & \\
\hline Increase $(>0.50)$ & & $72(15.1 \%)$ & --- & \\
\hline Normal $(0.4-0.50)$ & & $210(43.9 \%)$ & --- & \\
\hline Decrease $(<0.4)$ & & $196(41.0 \%)$ & --- & \\
\hline
\end{tabular}

* The number included cases with increased and normal PLT counts (They were not differentiated in the report).

a-Chi-square test. 
also isolated from L. scutellare, which was confirmed as the vector of scrub typhus in the study area $[19,33]$. The present study, together with our previous work $[19,32,33]$, suggests that the time when rodents cause maximum larval chigger infestation overlaps the period when the highest incidence of scrub typhus infections were reported in humans. Based on previous reports and this study, the main differences between the "summer type" and "autumn-winter type" scrub typhus in China were summarized in Table 6 .

Although certain clinical symptoms occurred more frequently in autumn-winter type cases than in summer type cases, regional lymphadenopathy, retro-orbital pain, electrocardiogram abnormity, hepatosplenomegaly, and flank tenderness were less common in the autumn-winter type cases. In contrast, some severe complications such as toxic myocarditis, alimentary tract hemorrhage, pleural fluid, or abdominal dropsy, commonly reported in the summer type cases in southern China [28], were not found at all in present study (Table 4). In addition, the abnormalities of WBC and PLT counts in present study were also less obvious than those reported for the summer type. The reports in southern China suggested that the percentages of cases with the increased and decreased
WBC counts were $32.9 \%$ and $27.0 \%$ respectively, which were higher than $\left(\chi^{2}=219.7, P<0.001\right)$ those reported in current study. Thrombocytopenia was reported in 51.1\% cases in southern China (Table 5) [28], also higher than $\left(\chi^{2}=222.7, P<0.001\right)$ that obtained in current study.

The reported percentages of eschar formation showed substantial variations across different studies [34-37], which could be explained in part by disparity of physicians experiences. Eschars could be detected relatively frequently on white-skinned Japanese children, however, it is relatively difficult to detect eschars on dark-skinned Thai pediatric cases [35-37]. Previous studies from some new endemic areas in northern China showed that the percentages of scrub typhus cases with eschars were $15 \%$ [38], 34\% [39], 84\% [40] and 100\% respectively [18]. In our study, $88.5 \%$ of cases had eschars. This is relatively high compared to other reports. Part of the reason is that physicians in our study team have worked there for many years and were very familiar with eschars. In addition, in our study we carried out thorough body examination for each suspected scrub typhus case, which may have contributed to high detection rate of eschars.

Table 6: Key differences between "summer type" and "autumn-winter type" scrub typhus in China

\begin{tabular}{|c|c|c|c|}
\hline & The summer type & The autumn-winter type & References \\
\hline Seasonal distribution of human cases & $\begin{array}{l}\text { Between March and November with a } \\
\text { peak occurrence in the summer between } \\
\text { June and August }\end{array}$ & $\begin{array}{l}\text { Exclusively from September to December } \\
\text { with a peak occurrence in October }\end{array}$ & {$[5-7]$} \\
\hline Geographical distribution & $\begin{array}{l}\text { Endemic in southern China (south of the } \\
\text { Yangtse River), including Guangdong, } \\
\text { Hainan, Guangxi, Fujian, Zhejiang, Yunnan, } \\
\text { and Hunan province. }\end{array}$ & $\begin{array}{l}\text { Endemic in northern China (north of the } \\
\text { Yangtse River), including Shandong, } \\
\text { northern Jiangsu, Tianjing, Shanxi, Hebei, } \\
\text { and Henan province }\end{array}$ & {$[5-7]$} \\
\hline Key reservoir hosts & $\begin{array}{l}\text { Rattus losea, } R \text {. flavipectus, and Apodemus } \\
\text { agrarius }\end{array}$ & A. agrarius, Cricetulus triton, and $R$. norvegicus. & {$[6,7]$} \\
\hline Key vector chigger mites & Leptotrombidium deliense & L. scutellare & {$[5-8]$} \\
\hline Virulence of $O$. tsutsugamushi isolates & $\begin{array}{l}\text { More virulent, because: }(I) \text { Being tested } \\
\text { with mice, the median lethal dose }\left(\mathrm{LD}_{50}\right) \\
\text { values of } O \text {. tsutsugamushi isolates were } 10 \text { - } \\
5-10^{-8} ;(2) \text {. tsutsugamushi could be } \\
\text { successfully isolated from cases by normal } \\
\text { experimental mice without any treatment }\end{array}$ & $\begin{array}{l}\text { Less virulent, because: (I) Partial } O \text {. } \\
\text { tsutsugamushi isolates do not lead to death } \\
\text { of mice; }(2) \text { Being tested with mice, } L_{50} \\
\text { values of most } O \text {. tsutsugamushi isolates } \\
\text { were } 10^{-0.5}-10^{-3} ;(3) \text { In order to isolate } O \text {. } \\
\text { tsutsugamushi successfully from cases, the } \\
\text { experimental mice must be treated by } \\
\text { cyclophosphamide to suppress immunity }\end{array}$ & {$[5,7]$} \\
\hline Key serotypes & Karp type, Gilliam type, and Kato type & Gilliam type & {$[5-7,9-11, \mid 4,19]$} \\
\hline Key genotypes* & Karp type & Kawasaki type & {$[12-14,20-22]$} \\
\hline Clinical features and complications & $\begin{array}{l}\text { Cases have the common clinical features } \\
\text { of scrub typhus such as fever, cutaneous } \\
\text { rash, eschar and local lympadenopathy. } \\
\text { However, the associated complications of } \\
\text { this type scrub typhus were severe }\end{array}$ & $\begin{array}{l}\text { Cases also have the common clinical } \\
\text { features of scrub typhus such as fever, } \\
\text { cutaneous rash, eschar and local } \\
\text { lympadenopathy. However, the associated } \\
\text { complications of this type of scrub typhus } \\
\text { were less severe than those of the summer } \\
\text { type }\end{array}$ & {$[5-7,15-18,28]$} \\
\hline
\end{tabular}

\footnotetext{
* Note: In China, the genotypes of scrub typhus have not been systematically studied until now. Data about genotypes of scrub typhus provided in the Table were only based on the primary results obtained in a few of areas in China. Karp types were identified as the key genotypes of the summer scrub typhus in Guangdong, Fujian, Hainan province in southern China [12-14], while Kawasaki types were identified as the key genotypes of the autumn-winter type scrub typhus in Shandong, northern Jiangsu province in northern China [14,20-22]
} 
Irons et al (1947) reported that $45 \%$ of confirmed eschars in US army personnel were detected on the feet and legs. Perineum, inguinal area, and axilla were also the preferentially eschar-manifested areas [41]. Kim et al (2007) reported that among the 162 adult scrub typhus cases in southwestern area of Korea, most cases had eschars on the front of the body. Eschars were primarily detected in males within $30 \mathrm{~cm}$ below the umbilicus. Yet a different pattern was seen in females - the most prevalent area in females was the front chest above the umbilicus [42]. In present study, a similar distribution of eschar was seen for both males and females except that eschars were more frequently detected in front chest above umbilicus in females than in males (Table 4). The distribution of eschar on body surface might be associated with dressing styles and personal hygiene, as the two factors affect how and where chiggers entered and stayed on the body surface $[5,6,9]$. Ten eschars from 10 cases whose serum samples were IFApositive and blood samples were PCR-positive were also tested positive by PCR. This result suggested that eschar could be used as an alternative indicator for the diagnosis of O. tsutsugamushi infection [27]. In addition, O. tsutsugamushi DNAs were detected in all blood samples collected from 10 cases with eschar, indicating that the cases were bitten by scrub typhus infected chiggers.

Serodiagnosis methods of scrub typhus include Weil-Felix test, IFA, enzyme-linked immunosorbent assay (ELISA) and indirect immunoperoxidase (IIP). Weil-Felix test is neither sensitive nor specific, and replaced by IFA $[43,44]$. IFA is a well method for serodiagnosis of scrub typhus if it was conducted by skilled lab-persons. However, it always provides false positive results. Therefore, the real-positives might be lower than that obtained by IFA. ELISA and IIP are more accurate than the IFA [45-47], but the two methods were not commercially available in China. Thus IFA was still widely used in China for serodiagnosis of scrub typhus until now. In present study, the confirmatory serodiagnosis of scrub typhus was made in case of a fourfold or greater rise in titers between paired acute and convalescent sera, or IgM or IgG titer in a single serum above 1:80, or 1:400 [5,24-26]. However, a few of cases whose IgM or IgG titer in a single serum under $1: 80$, or 1:400 might be neglected by IFA test according to this diagnosis criterion, especially when their convalescent sera were unavailable. This is a limitation of the present study.

The Kawasaki strain was first isolated from Japanese scrub typhus cases by using cyclophosphamide-treated mice in Miyazaki prefecture, and it was then found to be widely distributed in other infected areas of Japan, such as Kyushu, Kanagawa prefectures $[48,49]$. Kawasaki strain was defined as a new type of $O$. tsutsugamushi distinguishable from prototype strains Gilliam, Karp, and Kato by antigenic analyses using polyclonal and monoclonal anti- bodies [49]. However, some degree of cross-reactivity existed between the 54- to 56 kilodalton polypeptides of Kawasaki and Gilliam strains in immunoblotting analyses [50]. Ohashi et al [51] also found that the sequence homology of scrub typhus antigen 56-kilodalton (Sta56) surface protein gene between Kawasaki and Gilliam strain was the highest among the known O. tsutsugamushi strains, and thus high cross-immunoreaction existed between the two antigens. Because Kawasaki strain was not available as antigen in China, we had to use Karp, Kato, and Gilliam strains as antigens for IFA. In present study, serotypes of IFA-positive sera were found to be the Gilliam type, while genotyping results of eschars, and isolates from some serologically confirmed cases suggested that the genotype to be similar to the Kawasaki strain. This inconsistency could be in part due to the existence of cross-immunoreaction between Kawasaki and Gilliam strain $[50,51]$, such that antibodies to Kawasaki strain in the sera of cases in northern China could also be tested positive by IFA when we used Gilliam strain as antigen instead of Kawasaki strain.

\section{Conclusion}

The study, for the first time, comprehensively summarizes clinical and epidemiologic characteristics of the autumnwinter type scrub typhus in China. This type of scrub typhus emerged in 1986 in northern China and occurred exclusively from September to December with an occurrence peak in October, which was different from the summer type endemic in southern China. In comparison with the summer type, complications associated with autumnwinter type scrub typhus were less severe, and abnormalities of routine hematological parameters were less obvious. Despite some limitations inherent in the present study, this 12-year study provides important information on clinical and epidemiologic features of the autumnwinter type scrub typhus. These findings provide valuable information in assisting physicians in the diagnosis, treatment, and prevention of the disease.

\section{Competing interests}

The authors declare that they have no competing interests.

\section{Authors' contributions}

YL carried out the study design, epidemiological investigation, and drafted the manuscript. JS, YX, GL, LL, HX, NJ, YG, HY, SZ, PZ, JM, PF, SM participated in the epidemiological investigation, and sample and data collection in the field. DF, ZZ, WC and SL participated in the study design and helped to draft the manuscript. YL, SL, WC conceived the study and coordinated all the activities. All authors read and approved the final manuscript.

\section{Acknowledgements}

We are grateful to Dr Guang-hua Wu and Dr Heng-bin Guo (Nanjing Medical Institute, Nanjing) for help isolating O. tsutsugamushi, and Dr Zhang Hai- 
lian (National Vaccine and Serum Institute, Beijing) for her kind help in identifying O. tsutsugamushi. We thank Dr Wen-yi Zhang for his help in preparing the figure. We also thank the physicians and staff of Wanggou, Fangcheng, Huyang, Xinqiao and Shangye township hospitals in Feixian county, Shandong province for filling questionnaires. SL is grateful to support from Public Health Preparedness for Infectious Diseases (PHPID) at The Ohio State University. This research was supported by the National Nature and Science Foundation of the People's Republic of China (No. 30872 I55).

\section{References}

I. Silpapojakul K: Scrub typhus in the Western Pacific region. Ann Acad Med Singapore 1997, 26:794-800.

2. Kawamura A, Tanaka $H$, Tamura A: Tsutsugamushi Disease. Tokyo: University of Tokyo Press; 1995.

3. Walker DH, Mattern WD: Rickettsial vasculitis. Am Heart J 1980, I 00:896-906.

4. Allen AC, Spitz S: A comparative study of the pathology of scrub typhus (tsutsugamushi disease) and other rickettsia diseases. Am J Pathol 1945, 2 1:603-680.

5. Chen XR: Scrub typhus and Orientia tsutsugamushi. Beijing: Military Medical Science Press; 200I.

6. Wu GH: The epidemiological characteristics and prevention and cure of scrub typhus in China. Chin J Public Health 2000, I 6:777-779.

7. Yu ES: Studies on scrub typhus in China. Hongkong: Asia Medicine Press; 2000.

8. $\mathrm{Li} \mathrm{JC}$, Zheng $\mathrm{XY}, \mathrm{Xi} \mathrm{ZY}$ : Review on the studies of chigger mites and scrub typhus in China. Chin J Public Health 2000, I 6:773-775.

9. Chen XR: Advancement in the study of pathogeny of Orientia tsutsugamushi in China. Chin J Public Health 2000, 16:775-777.

10. Zhang HL, Liu YY, Wang SS: Serotype and biological characteristics of Orientia tsutsugamushi in Guangxi province. Chin J Epidemiol 1989, 10:S76-78.

II. Chen YM, Feng XG, Lei YM: Serotype and biologicalcharacteristics of Orientia tsutsugamushi in Yunnan province. Chin J Zoon 1997, 13:13-15.

12. Zhang HH, Li JC, Zheng XY, Xi ZY: Amplification and identification of the gene encoding the type-specific antigen from Rickettsia tsutsugamushi . Acad I SUM S 1999, 20:20-22.

13. Peng GF, Wang SS, Jiang PL, Huang JL, Zeng NH, Cui H, Chen XR, Zhu SF, Wang ZB: Analyses of Orientia tsutsugamushi isolated in Nanpeng island by PCR/RFLP. Chin / Zoon 1999, 15:60-61.

14. Guo HB: Recent advancement in the study of serotype and genotype of Orientia tsutsugamushi in China. Chin J Zoon 2000, 16:79-8I.

15. Yang YF, Wang JL, Yao YC, Cheng ZR, Xu ZP, Wang M: Preliminary investigation of scrub typhus in Shandong Province. Chin J Epidemiol 1987, 8:280.

16. Su DM, Xu MH, Liu Y: Preliminary investigation of scrub typhus in Taidong county, northern Jiangsu Province. Chin J Epidemiol 1989, 10:65.

17. Huang $\mathrm{Y}$, Jiang $\mathrm{YH}$ : The prevalent status of tsutsugamushi disease in China. Chin Trop Med 2003, 3:62-63.

18. Zhang AM, Deng WB, Hao ZY, Lv JR, Song ZQ, Liang YH, Chen ML, Niu DS, Cui H, Yang RF: Serological epidemiological investigations on scrub typhus in Henan province, Mid-China. Henan J Prev Med 2006, I 7:6-7.

19. Liu YX, Yang ZQ, Wu QY, Sun HL, Meng XR, Peng ZL, Miao ZS: Epidemiological study of autumn-winter type scrub typhus in a new endemic focus of Fei County, Shandong Province, China. Syst Appl Acarol 2000, 5:25-31.

20. Guo HB, Pan SZ, Li XF, Tang JQ, Zhang Y, Wu GH: Studies on detecting and typing of Rickettsia tsutsugamushi by polymerase chain reaction. Chin I Zoon I997, I3:8-I I.

21. Liu YX, Gao Y, Zhao ZT, Zhang JL, Yang ZQ, Bu XP, Su J): Amplification and typing of Sta56 gene of Orientia tsutsugamushi from Shandong province. Chin I Epidemiol 2004, 25:698-70I.

22. Liu YX, Zhao ZT, Gao Y, Jia CQ, Zhang JL, Yang ZQ, Wang SM, Jiang BF: Characterization of Orientia tsutsugamushi strains isolated in Shandong Province, China by immunofluorescence and restriction fragment length polymorphism (RFLP) analyses. Southeast Asian J Trop Med Public Health 2004, 35:353-357.
23. The geography and climate database of Feixian county, Shandong province [http://cn.zhaoshang-sh.com/Shandong/Linyi/ LinyiTZHJ/I0179594.html]

24. Brown GW, Shirai A, Rogers C, Groves MG: Diagnostic criteria for scrub typhus. Probability values for immunofluorescent antibody and Proteus OX-K agglutinin titers. Am J Trop Med Hyg 1983, 32:1101-II07.

25. Chen HL, Shieh GJ, Chen HY, Horng CB: Isolation of Rickettsia tsutsugamushi from the blood samples of patients in Taiwan. J Formos Med Assoc 1995, 94:SII2-SII9.

26. World Health Organization (WHO): WHO-Recommended Surveillance Standards. Second edition. 2004 [http://www.who.int/ csr/resources/publications/surveillance/ WHO CDS CSR ISR 992 EN/en/].

27. Liu YX, Cao WC, Gao Y, Zhang JL, Yang ZQ, Zhao ZT, Foley JE: Orientia tsutsugamushi in eschars from scrub typhus patients. Emerg Infect Dis 2006, 12: I 109-1 I I2.

28. Zhou J, Li DC, Lu ZJ: Clinical features of tsutsugamushi disease among children and adults in north of Guangdong province. Acta Medicine Sinica 2006, 19:64I-643.

29. Bang HA, Lee MJ, Lee WC: Comparative research on epidemiological aspects of tsutsugamushi disease (scrub typhus) between Korea and Japan. Jpn J Infect Dis 2008, 6 I: | 48-I 50.

30. Ogawa M, Hagiwara T, Kishimoto T, Shiga S, Yoshida Y, Furuya Y, Kaiho I, Ito T, Nemoto H, Yamamoto N, Masukawa K: Scrub typhus in Japan: epidemiology and clinical features of cases reported in 1998. Am J Trop Med Hyg 2002, 67:162-165

31. Hashimoto S, Kawado M, Murakami Y, Izumida M, Ohta A, Tada $Y$, Shigematsu M, Yasui Y, Taniguchi K, Nagai M: Epidemics of vectorborne diseases observed in infectious disease surveillance in Japan, 2000-2005. J Epidemiol 2007, I 7:S48-55

32. Liu YX, Zhao ZT, Yang ZQ, Zhang JL, Xu JJ, Wu QY, Peng ZL, Miao ZS: Epidemiological studies on host animals of scrub typhus of the autumn-winter type in Shandong Province, China. Southeast Asian J Trop Med Public Health 2003, 34:826-830.

33. Liu YX, Wu QY, Yang ZQ, Sun HL, Meng XR, Su M, Zhang XL: Studies on $L$. scutellare as a vector of scrub typhus of the autumnwinter type in Shandong Province. Literature and Information on Preventive Medicine 1997, 3:305-306.

34. Silpapojakul K, Varachit B, Silpapojakul K: Pediatric scrub typhus in Thailand: a study of 73 confirmed cases. Trans $R$ Soc Trop Med Hyg 2004, 98:354-359.

35. Silpapojakul K, Chupuppakarn S, Yuthasompob S, Varachit B, Chaipak D, Borkerd T, Silpapojakul K: Scrub and murine typhus in children with obscure fever in the tropics. Pediatr Infect Dis J 1991, I0:200-203.

36. Satari HI, Brabin BJ, Samuel S, Tanskul P, Vasanawathana S: Scrub typhus in Thai children: clinical features and serological study. Abstract of a presentation at the 45th Ann. Mtg. of the American Society of Tropical Medicine and Hygiene, Baltimore, MD December I-5, 1996. Am J Trop Med Hyg 1996, 55(2 suppl):219.

37. Basnyat B, Belbase RH, Zimmerman MD, Woods CW, Reller LB, Murdoch DR: Clinical features of scrub typhus. Clin Infect Dis 2006, 42:I505-I506.

38. Feng $M Q$, Yang YD, Zhang $W$ : The first appearance of scrub typhus in eastern suburb of Jinan. Shandong Med 1989, 29:35.

39. Chen LB, Tian FJ, Sun T: The first discover of scrub typhus in Rencheng suburb of Jining, Shandong province. Chin J Zoon 1997, I 3:70-7|.

40. Liu DB, Xu XH, Mao YP: The epidemiological report of the first outbreak of scrub typhus in Laizhou County, Shandong province. Disease surveillance 1998, 13:470-47I.

41. Irons EN, Armstrong HE: Scrub typhus in Dutch New Guinea. Ann Intern 1947, 26:20I-220.

42. Kim DM, Won KJ, Park CY, Yu KD, Kim HS, Yang TY, Lee JH, Kim $\mathrm{HK}$, Song HJ, Lee SH, Shin H: Distribution of eschars on the body of scrub typhus patients: A prospective study. Am J Trop Med Hyg 2007, 76:806-809.

43. Bozeman FM, Elisberg BL: Serological diagnosis of scrub typhus by indirect immunoflorenscence. Proc Soc Exp Biol Med 1963, I | 2:568-673.

44. Kelly DJ, Wong PW, Gan E, Lewis GE Jr: Comparative evaluation of the indirect immunoperoxidase test for the serodiagnosis of rickettsial disease. Am J Trop Med Hyg 1988, 38:400-406. 
45. Kelly DJ, Wong PW, Gan E, Chye CT, Cowan D, Lewis GE Jr: Multilaboratory evaluation of a scrub typhus diagnostic kit. Am J Trop Med Hyg 1990, 43:30I-307.

46. Suto $\mathrm{T}: \mathbf{A}$ ten years experience on diagnosis of rickettsial diseases using the indirect immunoperoxidase methods. Acta Virol 1991, 35:580-586.

47. Coleman RE, Sangkasuwan V, Suwanabun N, Eamsila C, Mungviriya S, Devine P, Richards AL, Rowland D, Ching WM, Sattabongkot J, Lerdthusnee K: Comparative evaluation of selected diagnostic assays for the detection of IgG and IgM antibody to Orientia tsutsugamushi in Thailand. Am J Trop Med Hyg 2002, 67:497-503.

48. Yamamoto $S$, Kawabata N, Ooura K, Murata M, Minamishima $Y$. Antigenic types of Rickettsia tsutsugamushi isolated from patients with tsutsugamushi fever and their distribution in Miyazaki prefecture. J Jpn Assoc Infect Dis 1989, 63: I09-II7.

49. Yamamoto S, Kawabata N, Tamura A, Urakami H, Ohashi N, Murata M, Yoshida Y, Kawamura A: Immunological prosperties of Rickettsia tsutsugamushi, Kawasaki strain, isolated from a patient in Kyushu. Microbiol Immonol 1986, 30:6I I-620.

50. Furuya $Y$, Yamamoto S, Otu M, Yoshida Y, Ohashi N, Murata M, Kawabata N, Tamura A, Kawamura A: Use of monoclonal antibodies against Rickettsia tsutsugamushi Kawasaki for serodiagnosis by enzyme-linked immunosorbent assay. I Clin Microbiol I99I, 29:340-345.

51. Ohashi N, Nashimoto H, lkeda H, Tamura A: Diversity of immunodominant 56-kDa type specific antigen (TSA) of Rickettsia tsutsugamushi . J Biol Chem 1992, 267:12728-12735.

\section{Pre-publication history}

The pre-publication history for this paper can be accessed here:

http://www.biomedcentral.com/1471-2334/9/82/prepub

Publish with Biomed Central and every scientist can read your work free of charge

"BioMed Central will be the most significant development for disseminating the results of biomedical research in our lifetime. "

Sir Paul Nurse, Cancer Research UK

Your research papers will be:

- available free of charge to the entire biomedical community

- peer reviewed and published immediately upon acceptance

- cited in PubMed and archived on PubMed Central

- yours - you keep the copyright

Submit your manuscript here:

http://www.biomedcentral.com/info/publishing_adv.asp
BioMedcentral 